\title{
HIV is always with me: men living with perinatally acquired HIV and planning their families
}

\author{
Marisa I Echenique' \\ Rachel S Bookman' \\ Violeta J Rodriguez' \\ Richard P LaCabe' \\ JoNell Efantis Potter ${ }^{2}$ \\ Deborah L Jones' \\ 'Department of Psychiatry and \\ Behavioral Sciences, ${ }^{2}$ Department of \\ Obstetrics and Gynecology, University \\ of Miami Miller School of Medicine, \\ Miami, FL, USA
}

This article was published in the following Dove Press journal:

Open Access Journal of Contraception

21 June 2017

Number of times this article has been viewed

Correspondence: Deborah L Jones

Department of Psychiatry and Behavioral Sciences, University of Miami Miller School of Medicine, I 400 NW IOth Ave., Suite 404A, Miami, FL 33136, USA

Tel +l $3052432041 / 2188$

Email djones@med.miami.edu
Abstract: Once expected to not survive childhood, youth with perinatally acquired HIV (YPHIV) have now reached young adulthood and are of reproductive age and sexually active. Given the health impact of pregnancy among YPHIV, understanding reproductive decision making may inform preconception counseling strategies. Most literature regarding reproductive health among YPHIV focuses on women, overlooking one of the most important factors influencing the reproductive decision-making process, male sexual partners. This study examined attitudes, perceptions, and experiences of young men with perinatally acquired HIV (YMPHIV) regarding family planning and relationships, safer sex, disclosure, stigma, and psychological health. Participants ( $\mathrm{n}=21$ ) were YMPHIV aged 18-24 years recruited in Miami, Florida. Focus groups $(n=4)$ were conducted; qualitative data were analyzed using grounded theory. HIV disclosure, stigma, fertility intentions, safer preconception knowledge, attitudes and practices, family planning communication with medical providers and family, and mental health emerged as themes. Results suggest that despite accurate knowledge regarding healthy preconception practices, psychopathology, substance use, and stigma impact the uptake of HIV health care interventions. Effective interventions on preconception counseling may require more tailored approaches than knowledge-based psychoeducation alone, such as inclusion of psychological treatment, which could be offered in HIV health care settings to optimize health outcomes.

Keywords: preconception counseling, fertility decision making, young adults, HIV risk reduction, HIV knowledge

\section{Introduction}

Combination antiretroviral therapy has enabled perinatally HIV-infected individuals in the USA to mature to adulthood. As youth with perinatally acquired HIV (YPHIV) become sexually active, risk behaviors and associated adverse health outcomes become increasingly challenging. ${ }^{1}$ Most (89\%) YPHIV report that their first sexual encounter follows obtaining knowledge of their HIV status and some (26\%) report sexual debut prior to age $15 .^{2}$ As YPHIV reach reproductive age, family planning and reproductive health matters must be addressed by their caregivers and health care providers, given that most $(70 \%)$ report intentions to have children. ${ }^{2}$ Though unplanned pregnancy is common in the general population, ${ }^{3}$ the potential for negative health consequences among young adults with HIV and for neonates is high. YPHIV have been less likely to be sexually active, and those that were have been were more likely to have engaged in non-penetrative sex, ${ }^{4}$ though peer-group sexual activity may influence sexual activity. ${ }^{4}$ Despite the high-risk sexual behaviors and adverse health outcomes from an early age among both men and women, young men with perinatally acquired HIV (YMPHIV) 
are typically overlooked in HIV research. US racial/ethnic and sexual minority male youth aged 15-24 years have substantial sexual and reproductive health $(\mathrm{SRH})$ needs and are disproportionately impacted by sexually transmitted infections (STIs) and HIV. ${ }^{5}$ Rates of AIDS are greater in black and Hispanic youth than among whites, and efforts to provide psychoeducation about HIV, sexual risk behaviors, and reproductive health are needed for this population and their sexual partners. ${ }^{5-7}$

There is no established consensus on a standard for preconception care for males, though they could play an active role in fertility planning. ${ }^{8}$ Preconception counseling is designed to provide preconception health literacy, enhance preconception-focused communication with providers, and instill pregnancy-planning behaviors. However, in order to adequately provide preconception counseling to YMPHIV, it is critical to understand their unique circumstances associated with having a chronic, socially stigmatized, sexually transmissible infection. ${ }^{9}$ HIV has a pervasive impact on those living with the virus; sexual activity can generate anxiety about transmission, disclosure, loss of sexual spontaneity, and fear of rejection ${ }^{9-11}$ as well as psychiatric challenges associated with living with a highly stigmatized illness during a critical developmental stage like adolescence.

HIV-infected male youth have a high rate of psychiatric symptoms and behavioral problems, such as substance use and conduct disorders, and are twice more likely to meet criteria for attention-deficit/hyperactivity disorder, compared to HIV-negative youth from similar socioeconomic backgrounds. ${ }^{11-14}$ The presence of psychiatric illness may be associated with poor behavioral outcomes, such as risky sexual behaviors, and poses public health concerns regarding HIV transmission, unplanned pregnancy, and poor treatment adherence. ${ }^{11,15}$ Issues affecting the decision-making process include desires to have children, HIV health care, perceptions of health care provider attitudes regarding people with HIV having children, and HIV status disclosure. ${ }^{16}$ Given the potential health impact, understanding factors influencing the reproductive decision-making process of YMPHIV may provide guidance for preconception counseling strategies aimed at HIV treatment and prevention. ${ }^{9}$ Furthermore, there is need to focus on young men's perspectives regarding fatherhood, as well as their knowledge of risk-reduction strategies for childbearing. ${ }^{16,17}$

This study examined the experiences, attitudes, and perceptions among YMPHIV regarding desires to have children, family planning, relationships, safer sex, disclosure, stigma, and psychological health. It was hypothesized that understanding YMPHIV attitudes regarding preconception counseling and family planning and identifying factors that may influence HIV and reproductive care could be used to provide the foundation optimal health outcomes among YMPHIV.

\section{Methods}

The present study was qualitative and descriptive and was conducted using focus group discussions (FGDs). Participants were 21 YMPHIV aged 18-24 years. The sample size was determined using guidelines suggested in previous research for studies that planned to use grounded theory for analysis. ${ }^{18}$ Candidates were referred by staff at a University of Miami Clinic in Miami, Florida, which is one of the primary sites at which YPHIV receive health care from early infancy to adolescence. Referring clinic staff provided information on participant demographic characteristics (race, age, education level, relationship status, partner HIV status, and whether the participant was on antiretroviral), which were obtained by candidate self-report. The participant sample was representative of the clinic population, which consists of $\sim 60$ YMPHIV. No participants declined to participate. Study participants attended FGD at the study site, which was located at the investigators' laboratory; groups were a maximum of six persons per group. Two of the FGD had 6 participants, one 5, and one 4 participants; FGDs were conducted from January 30, 2015 , to June 15,2015 . Groups with four to six participants are referred to as mini-FDGs and may be used with "hard to reach" population subgroups, ${ }^{19}$ such as YMPHIV. Mini-FGDs allowed participants ample time to express their opinions and views and facilitated a more informal, conversational group setting to increase participants' level of comfort in discussing potentially sensitive topics and assessing individual-level perspectives. ${ }^{20}$ All participants provided written informed consent prior to participation in the study and were compensated $\$ 40$ for their time and transportation. FGDs were structured and led by a peer educator with extensive experience working with YPHIV. The FGD stems consisted of a set of open-ended questions designed to elicit information on attitudes, knowledge, and practices regarding conception, including communication with providers and partners (Table 1); all FGDs were recorded. Participants reporting a history of self-injury and suicidal intent were assessed for current/active suicidal ideation and intent to self-harm and provided with resources for outpatient care as needed; no participants were identified to be at immediate risk of selfharm or suicide. Also, the investigators were licensed clinical psychologists available during all participant interactions 
Table I Focus group discussion stems and questions

I. Fertility intentions, planning

a. What do you know about women who are HIV-positive who want to have children?

b. Do you think that most women want to have children? What kinds of things are women concerned about when it comes to having children?

c. Do women talk to men about wanting to have children?

d. What kinds of things do women talk to men about when they want to become pregnant?

e. Do you think most pregnancies are planned or accidental? Why?

f. Have you ever discussed with women what they do when they want to get pregnant? Do they ever talk to you about switching or stop using contraception when they want to get pregnant?

2. Knowledge and attitudes about safer conception practices

a. What do you know about men being concerned about getting HIV if their partner has HIV and wants to get pregnant?

b. What do men and women do to prevent HIV infection of the man if they want to have a baby?

c. What do men and women know about safe ways for the woman to become pregnant without risking infecting the man with HIV?

d. How do men and women choose a safer method for the woman to become pregnant? By safer we mean one that might protect the man from HIV infection.

e. How do men and women feel about these kinds of methods?

3. Safer conception counseling experiences with health care providers

a. What do you know about men coming to women's clinics prior to or during pregnancy?

b. What have you heard about men discussing pregnancy at women's appointments?

c. Do doctors and nurses ask women with HIV about their desires to get pregnant?

d. Which ones talk about it? What kind of doctors or nurses?

e. What kinds of suggestions do they make about getting pregnant?

4. Perceptions and interpretations of the counseling received with providers

a. What kinds of attitudes do doctors or nurses have about HIV-positive women getting pregnant?

b. How supportive or not supportive are doctors of women's plans for having children?

5. HIV disclosure, partner status, intimate partner violence (IPV)

a. Do you think most HIV-positive women share their status with their partners? Have you ever talked to your partners about their HIV status?

b. Do men share their status with women? Have you ever talked to your partners about your HIV status?

c. What do you know about men forcing unprotected sex or trying to get women pregnant?

d. Why do you think some women do not share their HIV status? Do you think they fear domestic violence?

6. Stigma

a. How does HIV stigma affect discussions with others about getting pregnant?

7. Influences of other persons in the men's social networks (e.g., family, mothers-in-law, close relatives, friends)

a. How supportive or not supportive are families about people with HIV getting pregnant?

b. How supportive or not supportive are friends about people with HIV getting pregnant?

8. Perceived cultural mores regarding childbearing

a. What does your culture say about HIV-positive women having babies or getting pregnant?

b. What do religious beliefs say about HIV-positive women having children or family planning?

9. Structural barriers

a. Have you ever heard about women having problems getting health care prior to or during pregnancy?

b. Do you think women continue to go to the doctor after the baby is born? Have you heard of men going with them?

for additional assessment, if needed. Study procedures were approved by the University of Miami Institutional Review Board prior to the onset of recruitment.

\section{Descriptive and qualitative analyses}

Demographic data from the referral site were analyzed using univariate statistics. FGD recordings were transcribed using participant pseudonyms and coded using grounded theory, ${ }^{21-24}$ systematically reviewing the data for emergence of conceptual categories. Coders used NVIVO9 software to review the transcripts. Data analysis began with open-coding to capture maximum detail and complexity, followed by a process of continual comparison and revision, with initially broad codes becoming more focused; additional themes arising were added to the coding classification scheme. Initial coding was conducted by three staff members who duplicate coded the first FGD and then reviewed their coding with each other for consistency between coders. When inter-rater discrepancies emerged, relevant passages were reviewed by the study team before a final code was assigned. Once the coding classification system was finalized, a structured codebook was developed. The codebook included definitions for the classification codes and coding guidelines with illustrative examples for each code. Given the low number of participants in each focus group, individual-level perceptions were captured, allowing calculation of counts and percentages 
for reporting. Using the final coded data, key themes were extracted and illustrative quotes were selected. A summary of the final themes is presented in Table 2 .

\section{Results}

All YMPHIV participating in this study were African American and had a mean age of 21 years $(S D=2)$. Participants were in high school or had completed high school, and none were married, though the majority (90\%) had girlfriends or steady partners. All of the participants' partners were initially HIVnegative; however, two reported that their girlfriends became infected with HIV during their relationship. All participants had been prescribed antiretroviral therapy, the majority $(80 \%)$ were currently taking medication; the remainder were not currently on medication due to associated side effects or other illnesses. The following participant quotes are provided using pseudonyms to enhance clarity.

\section{HIV disclosure and stigma}

Most issues related to relationships, pregnancy planning, and sexual practices appeared to be influenced by disclosure of HIV serostatus and stigma. Most participants $(80 \%)$ reported fearing "reactions and rejections" and were sensitive to being stigmatized against by sexual partners, families, friends, coworkers, and pastors. All participants were selective with whom they shared their status, and the majority $(88 \%)$ did not routinely disclose their HIV status to their partner(s). One man shared, since his partner became infected, he "feel like [he] murdered somebody ... feel like [he] killed her." Participants mentioned different factors that they used to define what constitutes a relationship, which determined whether participants disclosed their HIV status. Physical characteristics, trust, length of relationship, and how well they knew their partner were factors they considered when deciding whether to disclose their HIV status and engage in protected or unprotected sex. While all agreed that support from friends, families, and partners helped participants living with HIV, disclosure of HIV status was risky:

...especially 'cause all these, these people go on social media and like you could tell someone, but in the long run if y'all break up or something they'll use that against you, talk bad about you like, these chick they evil. Like there's nobody, you can't trust any[body]. [Richard]

Most participants (95\%) learned of their status by the age of 15 years. For some, only a few years have passed since discovering their status and they feel pressure to share their HIV status with partners as they become sexually active. Many began the process of disclosure

...ask[ing] the question, like, how would you feel if my friend had HIV? [Michael]

Most participants (80\%) had not disclosed their HIV status to partners. Some asserted that they learned not to disclose from their parents,

It's the same way with [my] grandma, she just lost her husband, he was infected with HIV, too, but he didn't tell her about it... it's fear. [Michael]

Table 2 Summary of emerging themes

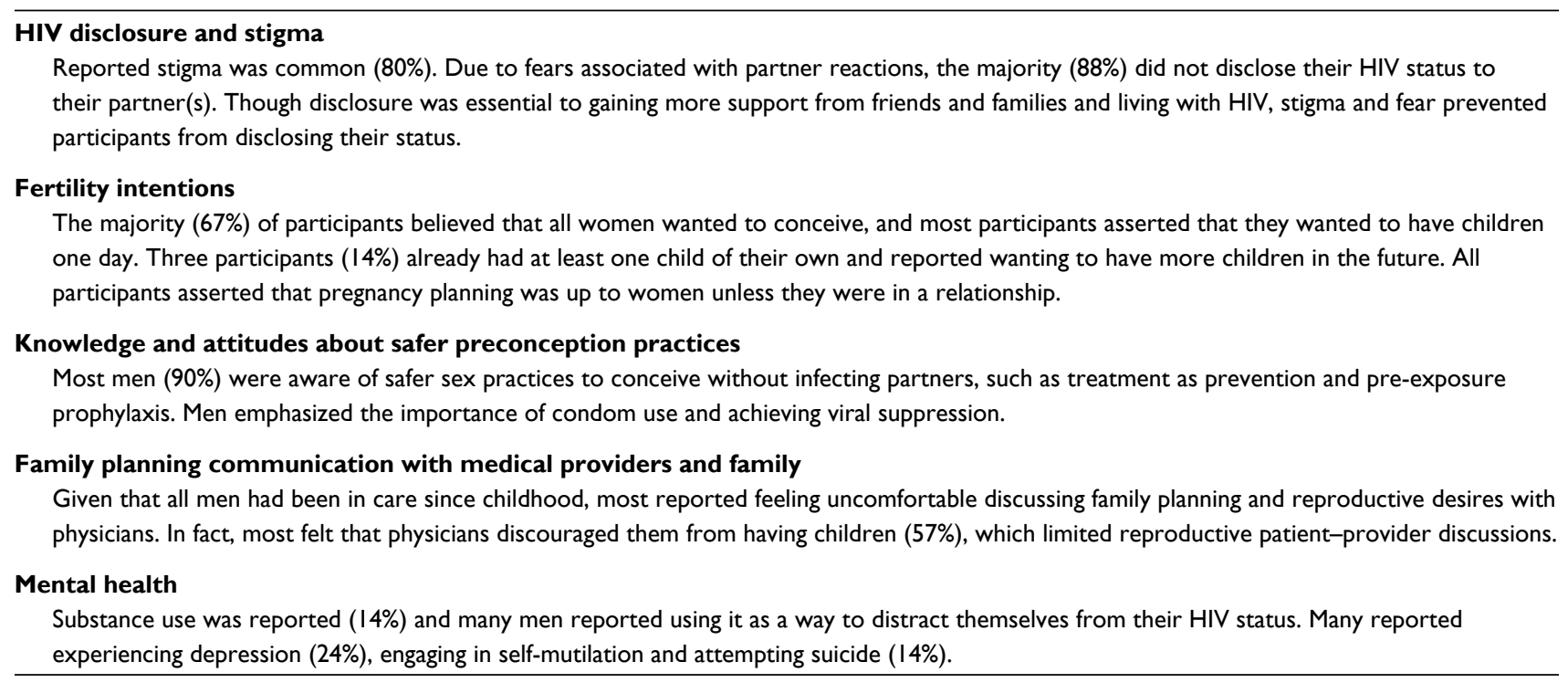


Though most participants reported not disclosing their HIV status to their partners, those who disclosed appeared to have different experiences and reactions from their partner upon disclosure. One participant shared that although his former partners in the past had rejected him after disclosing his HIV status, he had finally been able to find a partner who stayed after disclosing his status and appeared shocked by a positive reaction. This emphasized how stigmatizing beliefs may be internalized by participants, leading them to expect a negative reaction from potential romantic partners:

I pushed her off and like what's wrong and like "man I can't even do this" she like "why?" and check this out man I got this. If I tell you, I-I- - before I even disclose to let them know, let me know, I understand if you don't talk to me no more, but it's my right to tell you, you know what I mean? [Phillip]

I tell her everything. Then when a girl be like, they sit down like "okay, I'm a stay here." Like it's a meltdown to you like, damn I tell you everything, but you stay. [Phillip]

\section{Fertility intentions}

Three participants (14\%) already had at least one child of their own and reported wanting to have more children in the future. Among participants who did not have children, all but one (95\%) reported wanting children in the future. All participants who were not in a relationship asserted that pregnancy planning was up to women unless they were in a relationship. Participants shared negative and positive perceptions regarding women and pregnancy and how they perceived fatherhood:

Some women, they think ... "you do something for me to get you pregnant, I'm going to be with you" ... I'm stuck with you ... in reality, a guy can always leave, but the mother will always be there. The father can leave and have no feelings about it, like, "I didn't want this, you wanted it, so here." [Richard]

You know who stucked it in you know you knew yous coming you know? You know you was releasing in her so you gotta take the consequences too. You know? To be a father now. Cause some men want abortion and other girl don't want abortion so you just got to man up. [Michael]

Participants denied trying to force partners to have unprotected sex or try to "get them pregnant," but noted that women can "play hard to get."

\section{Knowledge and attitudes about safer preconception practices}

Most participants (90\%) were knowledgeable regarding safer sex practices; many were aware of strategies to become pregnant without infecting partners, e.g., pre-exposure prophylaxis (PrEP), treatment as prevention. Participants emphasized the importance of engaging in safer sex, agreeing on the importance of viral suppression.

[One method,] it's called PrEP. It's like when you want to have a baby, in order to have a HIV negative baby, both parties must adhere to the PrEP program. Before you get infected, you can take these medication if you feel are you in a ... high risk, you know, relationship, with one partner be positive, and that may help your partner or prevent your partner from being infected with the virus [...another method is] sperm washing. [Jarlin]

The main thing is use protection, and when you feel the time is right, sit down and talk to [your partner] and let them know what is going on with you. If they choose to leave, that means they really don't like you and they can't deal with the situation, which I don't blame them. Men and women feel if they disclose a partner will abandon them. A lot of men just don't care (about the consequences of non-disclosure of HIV status to sexual partners...) [Terry]

Men reported "most people do not use a condom all the time," citing fear of rejection, lack of HIV status disclosure, drug use, and mental illness as potential barriers to condom use. By not disclosing their HIV status, women were more accepting of engaging in unprotected sex. Men also reported that sometimes women pursued unprotected sex; and women who pursued having unprotected sex were perceived as wanting to force men into having children or already being infected with HIV:

I say I'm just not tryna have no kids forreal I be like 'cause sometime the girl can force herself on you and you could be telling them no, but like the boy could resist it more than the girl... [Terry]

If I had someone they know that I am positive and they don't want to use condoms I be thinking is something up with them too? Maybe they positive. And also there can be scenarios like a girl and you guys are doing whatever and you ain't got no protection and like she goes a little bit you know far and I'm trying you know like, chill. [Stephen] 


\section{Family planning communication with medical providers and family}

Men were "no strangers to doctors," having been under physicians' care throughout childhood due to their HIV diagnosis. Though participants typically did not attend discussions about pregnancy at women's appointments as they felt uncomfortable discussing the topic with physicians, about half (57\%) felt that physicians did not encourage them to have children and thereby attempted to influence their decision or ability to engage in preconception or family planning communication.

...no one tells, like, people that are HIV, not many people tell, that they should go get pregnant. Condoms are a common practice, but as medicines improve, doctors ... need to change their mindset along with these advancements. [Richard]

Some participants reported feeling as if doctors did not care about their patients, and even if they did, they did not care enough to initiate or have conversations about how HIV-affected couples can prevent infection to neonates or partners. Men reported that doctors' attitudes regarding conception were not always explicit - rather, sometimes they perceived it as a "negative vibe" (Andrew), an implicit bias that made patients feel as if pregnancy was something they should not do.

...I ain't meet one damn doctor yet that's cared. I mean let me help you man, let me show you what to do. [Andrew]

Men felt pressured by their family and partners to have children. Despite family pressure from their parents or grandparents, some men were hesitant about having children given that they believed it would be difficult to find someone they could disclose their HIV status to and have children with. Another participant shared that although his mother wanted him to have children and was excited about being a grandmother; his mother initially believed it was illegal for HIV-affected couples to conceive. As such, his mother feared that he would be prosecuted for having children as a man living with HIV:

My dad and my grandma want, she want, they want kids and I'm going to give them to em. It's like I gotta find the right person to disclose my status with. [Jarlin]

When I told my mom, you know, my girl's pregnant, she was scared for our life, she was scared I could go to jail ... I told this girl long time the first day we met about me, and we still together like, how am I going to go to jail? [Stephen]

\section{Psychological health}

A few participants (14\%) described substance use as a distractor from their HIV status. For others, coping mechanisms and responses included depression (24\%), self-injury, and attempted suicide (14\%). Past deliberate self-injury was reported as being motivated by both nonsuicidal and suicidal intents. In all instances, suicidal ideation and nonsuicidal self-injury were identified as being an escape from the pain and stigma participants experienced by knowing that they were infected with HIV:

My dad said, like, I got money for your funeral. We have it to bury you ... it hurts you know, but growing up I educated them, like listen, because back then I couldn't eat off a plate with the same spoon as them. That how real it was, it's like I use a spoon and throw that spoon away ... that bring stress to somebody. [Jarlin]

I tried ... I was going to cut my veins, but second time I was like this hurt - so. [Terry]

Most people like pain, like, I like pain. When I'm going through something I cut myself like 3 years ago cause like I wanted to commit suicide. If it wasn't for my doctor that actually read, you know, what I wanted to do and check my arm getting cut up, you know. I think, most people take you know knives to cut them. I take a key. I wanna feel the pain. [Sam]

I took a key. A car key and cut myself. I wanted to feel the pain 'cause I was hurting and nobody in my household don't know what I'm going through and all they saying is, do this, take this. [Evan]

\section{Discussion}

Given the potential health impact of pregnancy among YPHIV, understanding factors influencing the reproductive decision-making process is essential for appropriate HIV treatment and prevention. However, most of the literature regarding reproductive health in this population focuses on women, overlooking one of the most important factors influencing the reproductive decision-making process, male sexual partners. This qualitative study explored the experiences, attitudes, and perceptions of YMPHIV regarding having children, sexual practices, relationship dynamics, disclosure, and emotional health.

Overall, HIV-related stigma was an impediment to disclosure and clear communication regarding safer sex practices. Consistent with existing literature, young men appeared interested in eventually having children, ${ }^{16}$ and their families 
were generally supportive. Men were interested in avoiding unintentional pregnancies, citing the need to identify a person they could trust and to whom they could disclose their HIV status. Though men were familiar with strategies to avoid HIV transmission during conception, most had not discussed it with health care providers. Overall, young men reported similar psychological challenges to those previously described, encountering problems with depression, suicidality, and substance abuse. ${ }^{11,25}$ These psychological challenges were associated with having a highly stigmatized disease which reduced their willingness to disclose their serostatus to sexual partners.

The majority of men appeared to have adequate knowledge about HIV/STD transmission, HIV care, and treatment; in contrast, previous research found $47 \%$ felt uninformed about healthy preconception practices. ${ }^{26}$ Men appeared to have basic information on transmission and prevention, though this knowledge did not translate to practice of safer sex. The gaps between knowledge, intention, and actual behavior have been well documented, and previous studies suggest that perceived threat may influence the probability of adopting a behavior that may otherwise be beneficial. ${ }^{27}$ It is possible that despite having adequate knowledge and an intention to apply it, men may not have felt that these risk behaviors posed a direct threat to them; this perspective may be a function of youth and should be explored in future research. Men appeared doubtful or to distrust discussion of family planning with health care providers and noted that providers were unlikely to raise the topic during clinical care and did not condone them having children. These findings contrast with previous research, which suggests that providers who treat YPHIV may form close relationships with YPHIV and may be particularly supportive of YPHIV transitioning into adult clinics. ${ }^{28,29}$ Most men in this study had been attending the clinic their entire lives and could have viewed providers as parental figures, such that discussions of sexual practices were uncomfortable or could have been considered inappropriate or to stimulate disapproval. Nevertheless, findings from this study suggest that men's perceptions of the patient-provider relationship may have acted as barriers to discussing family planning, thereby increasing the risk of unplanned pregnancy and transmission of HIV. Results also suggest that while health care practitioners emphasize prevention messages, these strategies may not be sufficient to motivate behavior change due to emotional stress arising from the lack of HIV disclosure, psychological impairment, and substance use, and referrals for psychological treatment may be merited.
Communication between partners regarding family planning and having children was uncommon, as men perceived women to be deciding on their own if they wanted to conceive, and sometimes deliberately becoming pregnant. Men's distrust of their partners, and their own failure to disclose their HIV status, reduced the likelihood of engaging in discussions regarding conception and HIV prevention. Despite HIV-related knowledge, stigma and fear of rejection remained barriers to disclosure; fear of rejection was also associated with decreased condom use. Men expressed frustration with being stigmatized for an illness they had inherited which differed from the stigma of behaviorally acquired HIV. As previously found, YPHIV's perceived stigma may be related to the frequency of previous stigmatizing experiences, particularly with romantic partners who rejected them upon disclosure of HIV status, and this may have been the case for this specific sample of YMPHIV. ${ }^{30}$ Future interventions with this population should facilitate HIV status disclosure, target esteem building and coping strategies, and facilitate pathways to safer sexual practices.

\section{Implications}

While men in this study had the knowledge to maintain a healthy lifestyle and preconception practices, a variety of barriers may have reduced their ability or motivation to act upon this knowledge. Similarly, these barriers, e.g., mental illness, substance use, and stigma, impact the uptake of HIV health care interventions. Health care settings in which YPHIV receive routine HIV medical care and other services are an ideal setting to deliver preconception counseling and behavioral interventions. Effective interventions to reduce risk may require more tailored approaches than knowledgebased psychoeducation alone. Interventions in which psychosocial challenges are addressed may provide a framework for thinking about preconception practices and increase comfort, self-confidence, and competence and reduce barriers to the uptake of healthy conception practices.

\section{Limitations}

This pilot study utilized a small, convenience sample, and findings cannot be generalized to all YMPHIV. In addition, the impact of demographic variables and health status, e.g., CD4, viral load, on the determinants of sexual health and potential intervention approaches were not examined and could provide important information. Future research should explore these issues among larger samples. Finally, some questions addressed issues specific to female partners' 
attitudes and fertility intentions; future studies should more closely explore young men's own intentions.

\section{Conclusion}

This pilot study presented unique insights into the thoughts, feelings, and experiences of YMPHIV. Effective interventions on preconception counseling may require more tailored approaches than knowledge-based psychoeducation alone, such as inclusion of psychological treatment, which could be offered in HIV health care settings to optimize health outcomes in this vulnerable group.

\section{Acknowledgments}

This study was funded by grants from NIH, 5U01AI103397 and P30AI073961, and was made possible by support from the University of Miami Miller School of Medicine, UM SAC 2013-33.

\section{Disclosure}

The authors report no conflicts of interest in this work.

\section{References}

1. Murphy DA, Durako SJ, Moscicki AB, et al; Adolescent Medicine HIV/ AIDS Research Network. No change in health risk behaviors over time among HIV infected adolescents in care: role of psychological distress. J Adolescent Health. 2001;29(3):57-63.

2. Ezeanolue EE, Wodi AP, Patel R, Dieudonne A, Oleske JM. Sexual behaviors and procreational intentions of adolescents and young adults with perinatally acquired human immunodeficiency virus infection: experience of an urban tertiary center. $J$ Adolesc Health. 2006;38(6):719-725.

3. Finer LB, Zolna MR. Shifts in intended and unintended pregnancies in the United States, 2001-2008. Am J Public Health. 2014;104(Suppl 1):S43-S48.

4. Bauermeister JA, Elkington K, Brackis-Cott E, Dolezal C, Mellins CA. Sexual behavior and perceived peer norms: comparing perinatally HIV-infected and HIV-affected youth. $J$ Youth Adolesc. 2009;38(8):1110-1122.

5. Marcell AV, Morgan AR, Sanders R, et al. The socioecology of sexual and reproductive health care use among young urban minority males. J Adolescent Health. 2017;60(4):402-410.

6. Rangel MC, Gavin L, Reed C, Fowler MG, Lee LM. Epidemiology of HIV and AIDS among adolescents and young adults in the United States. J Adolescent Health. 2006;39(2):156-163.

7. Weintraub A, Mellins CA, Warne P, et al. Patterns and correlates of serostatus disclosure to sexual partners by perinatally-infected adolescents and young adults. Aids Behav. 2017;21(1):129-140.

8. Frey KA, Navarro SM, Kotelchuck M, Lu MC. The clinical content of preconception care: preconception care for men. Am JObstet Gynecol. 2008;199(6 Suppl 2):S389-S395.

9. Fernet M, Wong K, Richard ME, et al. Romantic relationships and sexual activities of the first generation of youth living with HIV since birth. AIDS Care. 2011;23(4):393-400.
10. Marhefka SL, Valentin CR, Pinto RM, Demetriou N, Wiznia A, Mellins CA. "I feel like I'm carrying a weapon." Information and motivations related to sexual risk among girls with perinatally acquired HIV. AIDS Care. 2011;23(10):1321-1328.

11. Mellins CA, Malee KM. Understanding the mental health of youth living with perinatal HIV infection: lessons learned and current challenges. J Int Aids Soc. 2013;16:18593.

12. Mofenson LM, Cotton MF. The challenges of success: adolescents with perinatal HIV infection. J Int Aids Soc. 2013;16:18650.

13. Mellins CA, Tassiopoulos K, Malee K, et al. Behavioral health risks in perinatally HIV-exposed youth: co-occurrence of sexual and drug use behavior, mental health problems, and nonadherence to antiretroviral treatment. Aids Patient Care STDS. 2011;25(7): 413-422.

14. Mellins CA, Brackis-Cott E, Leu CS, et al. Rates and types of psychiatric disorders in perinatally human immunodeficiency virus-infected youth and seroreverters. J Child Psychol Psychiatry. 2009;50(9): $1131-1138$.

15. Kacanek D, Malee K, Mellins CA, et al. Exposure to violence and virologic and immunological outcomes among youth with perinatal HIV in the pediatric HIV/AIDS cohort study. J Adolescent Health. 2016;59(1):30-37.

16. Fair $\mathrm{C}$, Wiener $\mathrm{L}$, Zadeh $\mathrm{S}$, et al. Reproductive health decision-making in perinatally HIV-infected adolescents and young adults. Matern Child Health J. 2013;17(5):797-808.

17. Rodriguez VJ, Spence A, Monda M, Potter J, Jones D. Couples Living with HIV: men's reproductive intentions, practices, and attitudes. $J$ Int Assoc Provid AIDS Care. 2017;16(2):133-139.

18. Mason M. Sample size and saturation in $\mathrm{PhD}$ studies using qualitative interviews. Forum Qual Soc Res. 2010;11(3).

19. Krueger RA, Casey MA. Focus Groups: A Practical Guide for Applied Research. Vol 3. London: Sage Publications; 2000.

20. Smith MW. Ethics in focus groups - a few concerns. Qual Health Res. 1995;5(4):478-486.

21. Glaser BG. Examples of Grounded Theory: A Reader. Mill Valley, CA: Sociology Press; 1993.

22. Glaser BG. More Grounded Theory Methodology: A Reader. Mill Valley, CA: Sociology Press; 1994.

23. Glaser BG. Theoretical Sensitivity: Advances in the Methodology of Grounded Theory. Mill Valley, Calif.: Sociology Press; 1978.

24. Glaser BG, Strauss AL. The Discovery of Grounded Theory: Strategies for Qualitative Research. London,: Weidenfeld and Nicolson; 1968.

25. Evangeli M, Foster C. Who, then what? The need for interventions to help young people with perinatally acquired HIV disclose their HIV status to others. Aids. 2014;28:S343-S346.

26. Sherr L, Barry N. Fatherhood and HIV-positive heterosexual men. HIV Med. 2004;5(4):258-263.

27. Braveman P, Gottlieb L. The social determinants of health: it's time to consider the causes of the causes. Public Health Rep. 2014; 129(Suppl 2):19-31.

28. Vijayan T, Benin AL, Wagner K, Romano S, Andiman WA. We never thought this would happen: transitioning care of adolescents with perinatally acquired HIV infection from pediatrics to internal medicine. AIDS Care. 2009;21(10):1222-1229.

29. Dowshen N, D'Angelo L. Health care transition for youth living with HIV/AIDS. Pediatrics. 2011;128(4):762-771.

30. Greenhalgh C, Evangeli M, Frize G, Foster C, Fidler S. Intimate relationships in young adults with perinatally acquired HIV: partner considerations. AIDS Care. 2013;25(4):447-450. 
Open Access Journal of Contraception is an international, peerreviewed, open access, online journal, publishing original research, reports, reviews and commentaries on all areas of contraception. In addition to clinical research, demographics and health-related aspects, the journal welcomes new findings in animal and preclinical studies relating to understanding the biological mechanisms and practical development of new contraceptive agents. The manuscript management system is completely online and includes a very quick and fair peer-review system. Visit http://www.dovepress.com/testimonials.php to read real quotes from published authors.

Submit your manuscript here: https://www.dovepress.com/open-access-journal-of-contraception-journal 